\title{
Papillary glioneuronal tumor growing slowly for 26 years: illustrative case
}

\author{
Kazuma Shinno, ${ }^{1}$ Yoshiki Arakawa, MD, PhD, ${ }^{1}$ Sachiko Minamiguchi, MD, PhD, ${ }^{2}$ Yukinori Terada, MD, PhD, ${ }^{1}$ Masahiro Tanji, MD, ${ }^{1}$ \\ Yohei Mineharu, MD, PhD, ${ }^{1}$ Takayuki Kikuchi, MD, PhD, ${ }^{1}$ Hironori Haga, MD, PhD, ${ }^{2}$ and Susumu Miyamoto, MD, PhD ${ }^{1}$ \\ Departments of ${ }^{1}$ Neurosurgery and ${ }^{2}$ Diagnostic Pathology, Kyoto University Graduate School of Medicine, Kyoto, Japan
}

BACKGROUND Papillary glioneuronal tumors (PGNTs) are classified as a type of World Health Organization grade I mixed neuronal-glial tumor. Most PGNTs involve cystic formations with mural nodules and solid components in the cerebral hemispheres, and PGNTs occur mainly in young adults. The long-term prognosis of PGNTs remains unclear.

OBSERVATIONS A 38-year-old male had been diagnosed with an arachnoid cyst associated with epilepsy in a local hospital. The initial magnetic resonance imaging (MRI) study showed the tumor as a heterogeneously enhanced nodule in the left postcentral gyrus. Subsequent MRI studies showed slow growth of the tumor for 26 years. He underwent gross total resection to control his epilepsy. The histopathological findings revealed pseudopapillary structures involving hyalinized blood vessels with a single or pseudostratified layer of cuboidal glial cells with round nuclei and scant cytoplasm. At the periphery of the lesion, Rosenthal fibers and acidophilic granule bodies were observed in the gliotic brain tissue.

Immunohistochemically, some interpapillary cells were positive for NeuN. On the basis of these findings, the tumor was diagnosed as a PGNT.

LESSONS This PGNT showed slow growth for 26 years. When recognizing a slowly growing tumor in the cerebral hemispheres of relatively young people that is associated with epileptic seizures, PGNT should be considered as a differential diagnosis.

https://thejns.org/doi/abs/10.3171/CASE21266

KEYWORDS papillary glioneuronal tumor; PGNT; slow growth; long clinical course; neuronal-glial tumor; epilepsy; seizure

Papillary glioneuronal tumors (PGNTs) are a type of rare benign mixed neuronal-glial tumor that occurs mainly in the cerebral hemispheres in young adults. PGNTs are clinically benign and rarely recur after gross total resection. However, diagnosing PGNTs with diagnostic imaging studies is not easy, and the long-term prognosis remains unclear. Here we report a case of PGNT associated with epileptic seizures. The PGNT gradually increased in size during 26 years of follow-up and was diagnosed after excision.

\section{Illustrative Case}

\section{Clinical History}

A 38-year-old male had experienced epileptic seizures with loss of consciousness at the age of 12 years old. He was diagnosed with an arachnoid cyst in his left parietal lobe by head computed tomography (CT) in a local hospital and was monitored. He subsequently exp- erienced repeated seizures without loss of consciousness. His epileptic seizures occurred once or several times per week and were more likely to happen during fatigue. His seizures started with numbness of the right leg that gradually progressed to the left lower and right upper limbs with weakness and inability to walk. At the age of 13 years, this patient presented with seizures with loss of consciousness again and underwent fenestration of the left parietal lobe lesion, but the frequency of his epileptic seizures did not decrease. His epilepsy was poorly controlled, and he was referred to our institute when he was 26 years old.

\section{Clinical Examinations}

Preoperative magnetic resonance imaging (MRI) studies showed the tumor $(14 \mathrm{~mm}$ ) with hypointensity in T1-weighted imaging (T1WI), hyperintensity in T2-weighted imaging (T2WI), and a heterogeneously

ABBREVIATIONS CT = computed tomography; ${ }^{18} \mathrm{~F}$-FDG PET/CT = ${ }^{18} \mathrm{~F}$-fluorodeoxyglucose positron emission tomography/computed tomography; FLAIR = fluid-attenuated inversion recovery; $\mathrm{Gd}$ = gadolinium; $\mathrm{MRI}$ = magnetic resonance imaging; $\mathrm{PFS}$ = progression-free survival; $\mathrm{PGNT}=$ papillary glioneuronal tumor; $\mathrm{T} 1 \mathrm{WI}=\mathrm{T} 1$-weighted imaging; $\mathrm{T} 2 \mathrm{WI}=\mathrm{T} 2$-weighted imaging .

INCLUDE WHEN CITING Published July 5, 2021; DOI: 10.3171/CASE21266.

SUBMITTED April 28, 2021. ACCEPTED May 5, 2021.

(C) 2021 The authors, CC BY-NC-ND 4.0 (http://creativecommons.org/licenses/by-nc-nd/4.0/). 
enhanced component in gadolinium-enhanced T1WI (Gd-T1WI) in the left parietal lobe. Fluid-attenuated inversion recovery (FLAIR) indicated brain edema around the tumor (Fig. 1A-D). ${ }^{18} \mathrm{~F}$-fluorodeoxyglucose positron emission tomography/CT ( $\left.{ }^{18} \mathrm{~F}-\mathrm{FDG} \mathrm{PET} / \mathrm{CT}\right)$ showed lower uptake in the lesion (Fig. 1E), and ${ }^{11} \mathrm{C}$-methionine PET/CT showed slightly higher uptake in the lesion, than in the normal brain parenchyma (Fig. 1F). The left parietal lobe lesion was thus described as a brain tumor, which also showed contrast during the 14 years of imaging follow-up, and the enhanced component increased in size over another 11 years (Fig. 2). Ganglioglioma, pleomorphic xanthoastrocytoma, oligodendroglioma, diffuse astrocytoma, and pilocytic astrocytoma were considered as differential diagnoses. Because the lesion was located near the right foot motor cortex and there was a risk of deficit by removing it, observation and additional anticonvulsants were selected at first. However, optimizing the dose of anticonvulsants failed to control his epileptic seizures sufficiently. Eventually, he underwent gross total resection to control his epilepsy. After excision, his epileptic seizures disappeared. Moreover, during follow-up 47 months after surgery, no epileptic seizures occurred, and no tumor recurrence was observed.

\section{Pathological Review of the Tumor Specimen}

Pathological findings indicated primarily pseudopapillary structures involving hyalinized blood vessels with a single or pseudostratified layer of small, round tumor cells with scant eosinophilic cytoplasm, without nuclear atypia (Fig. 3A and B). At the periphery of the lesion, eosinophilic granular bodies and Rosenthal fibers were also observed. Immunohistochemical studies indicated that many of the tumor cells were positive for glial fibrillary acidic protein and oligodendrocyte transcription factor 2 (Fig. 3C and D). Neuronal cells in the lesion were NeuN positive, and the intercellular space was positive for synaptophysin (Fig. 3E and F). Isocitrate dehydrogenase $1 \mathrm{R} 132 \mathrm{H}$ and p53 were both negative, and the Ki-67 labeling index was $<1 \%$. On the basis of these findings, the lesion was diagnosed as a World Health Organization (WHO) grade I PGNT.

\section{Discussion}

\section{Observations}

PGNT is a benign brain tumor with glial and neuronal components and occurs in the cerebral hemispheres predominantly in young adults. The disease concept was first proposed by Komori et al. in 1998. ${ }^{1}$ PGNT consists of pseudopapillary structures with few atypical astrocytes covering hyalinized blood vessels and small to large differentiated neurons that proliferate between those pseudopapillary structures. PGNT is classified as a type of neuronal and mixed neuronal-glial tumor in the WHO 2016 classification and is assigned WHO grade I. ${ }^{2}$ Most patients with PGNTs have a good prognosis. Approximately 130 cases have been reported in the current English-language literature. ${ }^{3}$ PGNT tends to occur in the temporal lobes of the cerebral hemispheres in $97 \%$ of reported cases. $^{1,4}$ PGNTs can sometimes develop in the cerebral ventricles. ${ }^{5}$ PGNTs are thought to be more likely to recur in cases in which the tumor is large in diameter and composed only of solid components. ${ }^{4}$ The present case also showed an enlarged nodule of the tumor. Imaging findings illuminated a high percentage $(91.8 \%)$ of cystic lesions with mural nodules and solid components. ${ }^{4} \mathrm{MRI}$ revealed that the nodule components showed low signal intensity, a
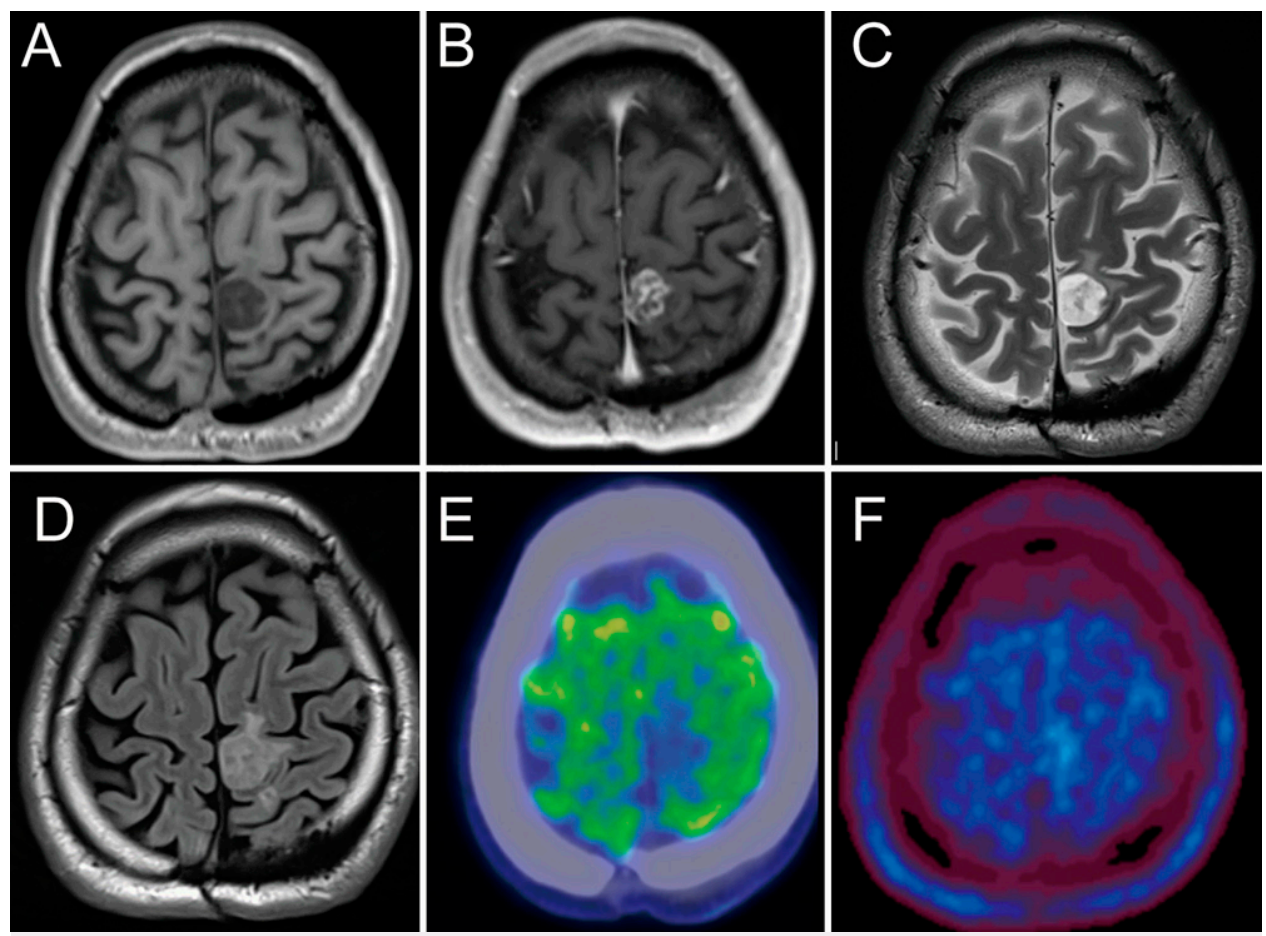

FIG. 1. A: T1Wl indicating a nodular tumor with hypointensity in the left parietal lobe. B: Gd-T1WI showing a heterogeneous enhanced component. C: T2WI showing the well-circumscribed tumor with hyperintensity. D: FLAIR indicating brain edema around the tumor. E: ${ }^{18} \mathrm{~F}-\mathrm{FDG}$ PET/CT showing the tumor with low uptake compared with normal brain parenchyma. F: ${ }^{11} \mathrm{C}$-Methionine PET/CT showing the tumor with slightly intense uptake. 

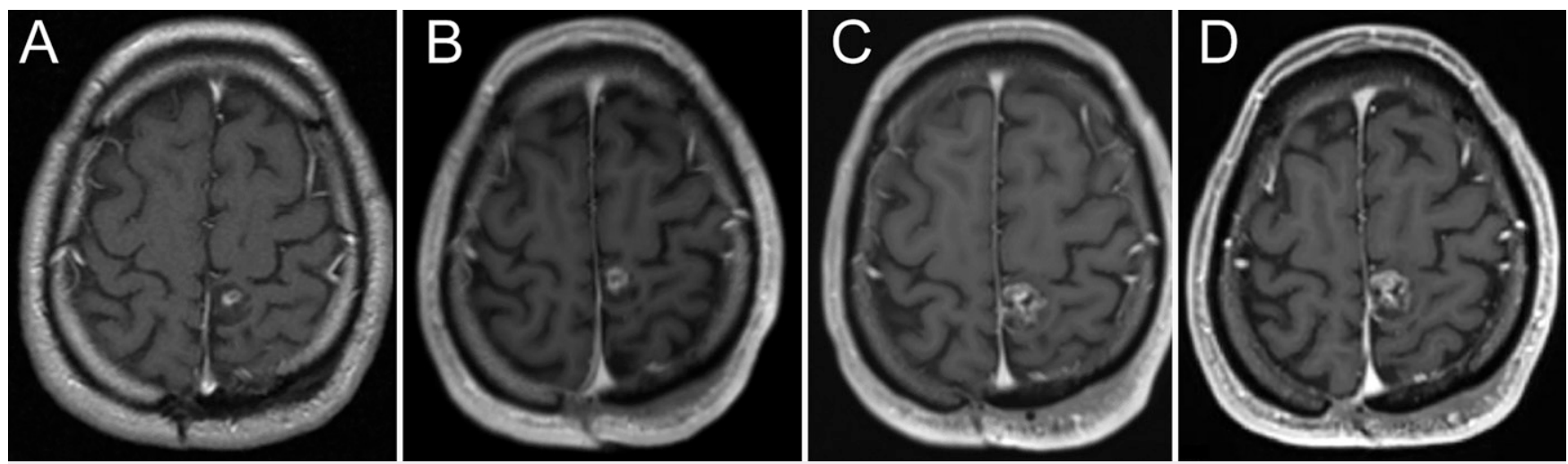

FIG. 2. A-D: Gd-T1WI scanned at 0, 6, 10, and 11 years after the patient first visited our institute, respectively (i.e., 15, 21, 25, and 26 years after the first diagnosis in a local hospital). The small solid tumor with a small enhanced component was observed in the left parietal lobe in (A). The lesion increased from $14 \mathrm{~mm}$ in diameter to $17 \mathrm{~mm}$ after 11 years of follow-up in (B-D). The tumor grew slowly, resulting in the expansion of edema.

gadolinium-enhancing effect on T1WI, and high signal intensity on T2WI and FLAIR sequences. PGNTs are sometimes associated with hemorrhagic onset and calcification, and primary metastatic dissemination was observed in 1 of a total of 71 cases. ${ }^{4}$ PGNTs are thought to frequently arise in supratentorial areas and may

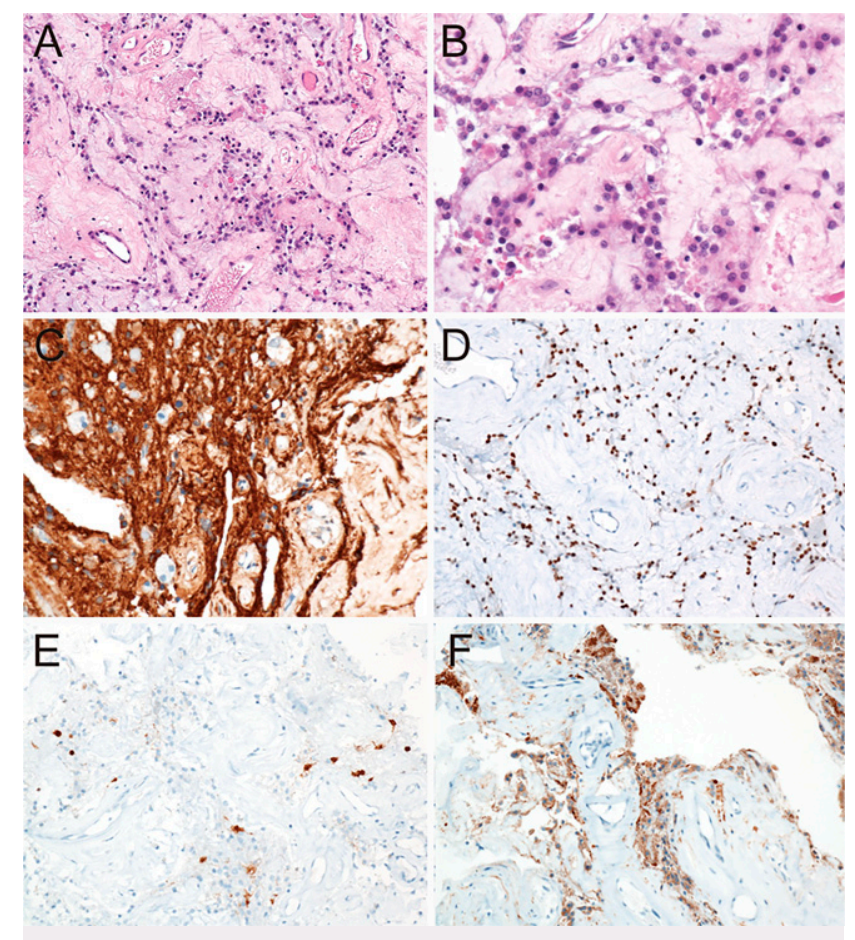

FIG. 3. A: The tumor showed papillary structures with hyalinized vessels (hematoxylin and eosin [H\&E] stain, original magnification $\times 20$ ). B: The tumor cells had round nuclei with eosinophilic scanty cytoplasm, without nuclear atypia $(H \& E$ stain, original magnification $\times 40)$. C: Glial fibrillary acidic protein (GFAP) was strongly expressed in the tumor cells (GFAP stain, original magnification $\times 20$ ). D: Oligodendrocyte transcription factor 2 (Olig2) was positive in the nuclei of interpapillary tumor cells (Olig2 stain, original magnification $\times 20$ ). E: NeuN positivity was found in some interpapillary cells (NeuN stain, original magnification $\times 20$ ). F: Synaptophysin was expressed in the cytoplasm of neuronal cells (synaptophysin stain, original magnification $\times 20$ ). cause epileptic seizures. Considering the present case, epileptic seizures were well controlled after gross total resection, and no postoperative seizures were reported. ${ }^{4}$

Regarding clinical symptoms, intracranial hypertension associated with cystic enlargement most frequently results in headache, nausea, and vomiting in about $60 \%$ of cases. Epileptic seizures are the second most common clinical manifestation, occurring in $32.8 \%$ of patients. ${ }^{4}$ Hemorrhage onset and neurological deficits are observed on rare occasions. ${ }^{3,6}$ The first-choice treatment for PGNT is total tumor excision, although relapse sometimes happens after resection. In most cases, the prognosis is excellent even with incomplete resection. ${ }^{4}$

A meta-analysis of PGNTs conducted in 2014 reported that $80 \%$ of patients underwent complete tumor resection; two received adjuvant radiation therapy after complete tumor resection; and six patients who underwent incomplete tumor resection also underwent adjuvant radiotherapy (two cases), chemotherapy (one case), or both (three cases). ${ }^{4}$ Furthermore, this analysis reported a 1.5-year progression-free survival (PFS) of $86 \% \pm 5 \%$ of about 56 cases (2-year PFS was $82 \% \pm 6 \%$ ). ${ }^{4}$ In 2015 , Park et al. reported 18 cases of atypical profiles with a Ki-67 index of $>5 \%$, which indicated the capacity for cell proliferation. Half of them were progressive or recurrent. No recurrence or progression was reported in cases in which the $\mathrm{Ki}-67$ index was $<5 \%{ }^{7}$ The median $\mathrm{Ki}-67$ index (proliferation index) is thought to be about $2 \%{ }^{4}$

PGNTs have a benign prognosis on the whole but are thought to feature a low 2-year PFS and a high risk of recurrence if the size of the tumor is $>3.5 \mathrm{~cm}$ and it predominantly consists of nodule lesions. ${ }^{4}$ In the present case, the tumor was $<3.5 \mathrm{~cm}$ in diameter and exclusively solid. However, it was accompanied by an enhanced portion over the 14-year course and showed a gradual increase in the enhanced portion during an additional 11 years of follow-up.

Although PGNT is a rare tumor, it should be differentiated from other glioneuronal tumors by keeping in mind the risk of growth and recurrence. The rate of growth of this tumor is slow, but it should be considered a possible cause of epileptic seizures, as observed in this case. Confirming the diagnosis of PGNT may be difficult, but gross total tumor resection could be an option in patients with poorly controlled epileptic seizures.

\section{Lessons}

We report a patient with PGNT that developed along with epilepsy and showed slow enlargement over a follow-up period of 26 years. 
PGNT should be considered as a differential diagnosis when a neoplastic lesion gradually grows, involves cysts with mural nodules and solid components, and is found in cerebral hemispheres of relatively young people.

\section{References}

1. Komori T, Scheithauer BW, Anthony DC, et al. Papillary glioneuronal tumor: a new variant of mixed neuronal-glial neoplasm. Am J Surg Pathol. 1998;22(10):1171-1183.

2. Louis DN, Perry A, Reifenberger G, et al. The 2016 World Health Organization Classification of Tumors of the Central Nervous System: a summary. Acta Neuropathol. 2016;131(6):803-820.

3. Rocka S, Neverauskiene L, Nelson EL, Burneikiene S. Papillary glioneuronal tumour: a case report. Cureus. 2019;11(3):e4215.

4. Schlamann $A$, von Bueren $A O$, Hagel $C$, et al. An individual patient data meta-analysis on characteristics and outcome of patients with papillary glioneuronal tumor, rosette glioneuronal tumor with neuropillike islands and rosette forming glioneuronal tumor of the fourth ventricle. PLoS One. 2014;9(7):e101211.

5. Demetriades AK, Al Hyassat S, Al-Sarraj S, et al. Papillary glioneuronal tumour: a review of the literature with two illustrative cases. $\mathrm{Br} \mathrm{J}$ Neurosurg. 2013;27(3):401-404.

6. Fujita Y, Kinoshita M, Ozaki T, et al. Enlargement of papillary glioneuronal tumor in an adult after a follow-up period of 10 years: a case report. J Surg Case Rep. 2018;2018(6):rjy123.

7. Park CK, Phi JH, Park SH. Glial tumors with neuronal differentiation. Neurosurg Clin N Am. 2015;26(1):117-138.

\section{Disclosures}

Dr. Arakawa reports grants from Siemens, Philips, Sanofi, Nihon Medi-Physics, MitsubishiTanabe, Takeda, Stryker, Astellas Pharma, Taiho Pharma, Pfizer, Brainlab, Merck, Chugai, Eisai, Meiji Seika, Daiichi Sankyo, Zeiss, Ono Pharmaceutical, and CLS Behring all outside the submitted work, and personal fees from Nippon Kayaku, AbbVie, Novocure, UCB Japan, Otsuka, Brainlab, Merck, Chugai, Eisai, Meiji Seika, Daiichi Sankyo, Zeiss, Ono Pharmaceutical, and CLS Behring all outside the submitted work.

\section{Author Contributions}

Conception and design: Arakawa, Shinno. Acquisition of data: Arakawa, Shinno, Haga. Analysis and interpretation of data: Shinno. Drafting the article: Arakawa, Shinno, Minamiguchi. Critically revising the article: Arakawa, Mineharu, Kikuchi, Miyamoto. Reviewed submitted version of manuscript: Arakawa, Minamiguchi, Terada, Tanji, Kikuchi. Approved the final version of the manuscript on behalf of all authors: Arakawa. Administrative/ technical/material support: Arakawa, Minamiguchi. Study supervision: Miyamoto.

\section{Correspondence}

Yoshiki Arakawa: Kyoto University Graduate School of Medicine, Kyoto, Japan. yarakawa@kuhp.kyoto-u.ac.jp. 